\title{
Propagation in Nonuniform Gyrotropic Media ${ }^{1}$
}

\author{
Stanley H. Gross
}

\begin{abstract}
Airborne Instruments Laboratory Division of Cutler-Hammer, Inc., Long Island, N.Y.
\end{abstract}
\author{
Leopold B. Felsen
}

Department of Electrophysics, Polytechnic Institute of Brooklyn, Brooklyn, N.Y.

(Received September 8, 1964; revised September 24, November 4, 1964)

\begin{abstract}
With the exception of the stratified layer approximation, the problem of wave propagation due to localized sources in nonuniform magneto-ionic media has received little attention. In previous work, the coupling between natural modes of the homogeneous gyrotropic medium has restricted the useful range of the analysis to the slowly varying case. In the present investigation, an $E$ and $H$ mode representation is used which results in a type of coupling independent of the rate of variation in the medium, thereby permitting the study of regions with strong inhomogeneities along the gyrotropic axis.

The coupling between the $E$ and $H$ modes now arises due to the deviation of the gyromagnetic parameter $Y=\omega_{c} / \omega$ from zero or infinite values. The uncoupled problems are therefore associated with the nongyrotropic $(Y=0$ or $Y=\infty)$ but nonuniform cases, and the procedure converges rapidly when $Y$ is large or small, even for rapid spatial variations of the medium parameters.

An iterative expansion is employed to deduce the gyrotropic corrections, and the procedure is phrased systematically in transmission line form. The analysis is applied to the evaluation of the far field radiated by an electric current element exterior to a semi-infinite, nonuniform plasma region.
\end{abstract}

\section{Introduction}

A fundamental difficulty in the analysis of wave propagation in a continuously stratified magneto-ionic medium is due to the coupling among the four wave types (upgoing and downgoing, ordinary and extraordinary) which may propagate independently when the medium is homogeneous. Four coupled first-order differential equations may be derived for the specification of the wave functions [Budden, 1961; Clemmow and Heading, 1954] and while a formal exact solution may be found under special conditions [H. Keller, 1953], the determination of explicit results has generally been based on perturbation techniques which converge rapidly when the coupling is small. Such approximation procedures have utilized as unperturbed solutions the magneto-ionic waves in a homogeneous region, with the coupling produced by the variation of the medium parameters along the axis of stratification. While the coupling is minimized when the variation is small, thereby permitting an approximate analysis of the four equations for the wave functions [Clemmow and Heading, 1954], there exist regions even under these conditions wherein two or more wave types may interact strongly (for example, near reflection points). A modification may then be employed by which the problem is phrased generally in terms of two coupled second-order equations [Hougardy, 1961]; the two solutions of each equation (for example, upgoing and downgoing waves of one type) may then be strongly coupled, whereas, interaction between the two pairs is weak. The analysis in all of these considerations is built around the unperturbed solutions for the homogeneous case, thereby making slow variation an essential requirement.

\footnotetext{
'The material for this paper has been taken from the dissertation of one of the authors, S. H. Gross, submitted to the Faculty of the Polytechnic Institute of Brooklyn in partial fulfillment of the requirements for the degree of Doctor of Philosophy, 1964.
} 
An alternative viewpoint is employed in the present treatment where the unperturbed situation is one for which the magneto-ionic dielectric tensor has a diagonal representation. In a coordinate system oriented so that the $z$-axis coincides with the direction of the d-c magnetic field $B$, this occurs when $B=\infty$ (uniaxial case) and, of course, also when $B=0$ (isotropic case). If the uniaxial medium parameters vary only (but arbitrarily) along $z$, four uncoupled wave solutions may be derived; the coupling in this instance is due to finite, rather than infinite, values of $B$ (or of the gyrofrequency $\omega_{c}$ ). The present formulation is therefore useful for the study of the influence of strong gradients in the constitutive parameters provided, however, that $\omega_{c}$ is large in comparison to the applied frequency $\omega$ and the plasma frequency $\omega_{p}$. (The constraint on $\omega_{p}$ relative to $\omega_{c}$ is actually less severe, see sec. 2.3b.) While this regime may exhibit strong anisotropic effects, analogous considerations apply as well to the almost isotropic range where $\omega_{c}$ is small.

The analysis proceeds from a guided wave viewpoint by which the field is decomposed into $E$ and $H$ modes with respect to the direction of $B$. The modal amplitudes are found to satisfy conventional transmission line equations which are coupled by the off-diagonal term in the dielectric tensor. A solution of these equations is achieved by an iterative scheme which converges rapidly when $\omega_{c}$ is very large or small. The procedure is illustrated for the case of a transverse electric current dipole embedded in a magneto-ionic medium with $z$-dependent electron density, with field expressions derived for the unperturbed situation $\left(\omega_{c}=\infty\right)$ as well as the first gyrotropic correction, $0\left(1 / \omega_{c}\right)$. Explicit results are summarized for the case of a half-space with constant density gradient, with the source located in the exterior region.

A variety of other applications for $Y \gg 1$ and $Y \ll 1$ have also been studied in detail and will be submitted for publication in separate form. It may be mentioned that although the case $Y \ll 1$ pertains more directly to the ionosphere than $\mathrm{l} \gg 1$, the latter has been chosen for illustration, since even the unperturbed solution $(Y=\infty)$ may exhibit strong anisotropic effects.

\section{Formal Solution}

As mentioned earlier, the direction of nonuniformity and the steady magnetic field are taken along the $z$-axis of a right-handed Cartesian coordinate system, so that the configuration in the $x-y$ plane is uniform and infinite in extent. The relative permittivity tensor $\overline{\bar{\epsilon}}$ relating the electric displacement vector and the electric field intensity vector in the nonuniform, gyrotropic medium may be expressed as follows [Budden, 1961]:

$$
\overline{\bar{\epsilon}}=\left(\begin{array}{ccc}
\epsilon_{1} & -i \epsilon_{2} & 0 \\
i \epsilon_{2} & \epsilon_{1} & 0 \\
0 & 0 & \epsilon_{3}
\end{array}\right)
$$

where the double bar above the symbol denotes a tensor, the expression on the right is its matrix representation and the elements of the matrix $\epsilon_{1}, \epsilon_{2}$, and $\epsilon_{3}$ are permitted to be functions of $z$. The permeability of the medium is taken as that of free space. Throughout this paper the implied time dependence is taken as $e^{-i \omega t}$, where $\omega$ is the radian frequency of the source, and the steady magnetic field is taken along the negative $z$-axis.

The quantities $\epsilon_{1}, \epsilon_{2}$, and $\epsilon_{3}$ for a lossless gyrotropic plasma medium have the form [Budden, 1961]

$$
\begin{aligned}
\epsilon_{1} & =1-\frac{X}{1-Y^{2}} \\
\epsilon_{2} & =\frac{X Y}{1-Y^{2}} \\
\epsilon_{3} & =1-X
\end{aligned}
$$


where:

$$
\begin{aligned}
X & =\omega_{p}^{2} / \omega^{2} \\
Y & =\omega_{c} / \omega \\
\omega_{p} & =\text { plasma frequency }=\sqrt{\frac{N e^{2}}{\epsilon_{0} m}} \\
\omega_{c} & =\text { gyromagnetic frequency }=\left|\frac{e B}{m}\right|, \\
B & =\text { steady magnetic field, }
\end{aligned}
$$

$\epsilon_{0}$ is the permittivity of free space, $e$ and $m$ are the electronic charge and mass and $N$ is the electron density of the medium.

\subsection{Reduction of the Maxwell Field Equations}

It has been shown elsewhere [Arbel and Felsen, 1963] that the Maxwell equations for the transverse field components may be written as follows:

$$
\left.\begin{array}{l}
-\frac{\partial \bar{H}_{t}}{\partial z}=-i \omega \epsilon_{0}\left(\overline{\bar{\epsilon}}_{t}+\frac{1}{k^{2}} \nabla_{t} \nabla_{t}\right) \cdot \bar{z}_{0} \times \bar{E}_{t}+\bar{z}_{0} \times \hat{\bar{J}}_{t} \\
-\frac{\partial \bar{E}_{t}}{\partial z}=i \omega \mu_{0}\left(\overline{\overline{1}}_{t}+\frac{1}{k^{2} \epsilon_{3}} \nabla_{t} \nabla_{t}\right) \cdot \bar{z}_{0} \times \bar{H}_{t}-\bar{z}_{0} \times \hat{\bar{M}}_{t}
\end{array}\right\}
$$

where the subscript $t$ is used to designate vector components transverse to $z, \nabla_{t}=\nabla-\bar{z}_{0} \frac{\partial}{\partial z}$, $k^{2}=\omega^{2} \mu_{0} \epsilon_{0}, \bar{E}$ and $\bar{H}$ are the electric and magnetic field intensity vectors, $\mu_{0}$ is the permeability of free space, $\overline{\bar{l}}_{t}$ is the transverse unit dyadic, $\bar{z}_{0}$ is the unit vector along $z$, and $\overline{\bar{\epsilon}}_{t}$ is the transverse tensor givey by:

$$
\overline{\bar{\epsilon}}=\left(\begin{array}{cc}
\epsilon_{1}-i \epsilon_{2} \\
i \epsilon_{2} & \epsilon_{1}
\end{array}\right)
$$

Furthermore,

$$
\begin{gathered}
\hat{\bar{J}}_{t}=\bar{J}_{t}-\frac{1}{i \omega \mu_{0}} \nabla_{t} M_{z} \times \bar{z}_{0}, \\
\hat{\bar{M}}_{t}=\bar{M}_{t}+\frac{1}{i \omega \epsilon_{0} \epsilon_{3}} \nabla_{t} J_{z} \times \bar{z}_{0},
\end{gathered}
$$

are equivalent transverse source distributions whose values are expressed in terms of those of the specified electric and magnetic vector current densities $\bar{J}$ and $\bar{M}$, respectively. The $z$ components of the field vectors, $E_{z}$ and $H_{z}$, may be expressed in terms of the transverse field components and the $z$ components of the applied current densities [Arbel and Felsen, 1963].

To solve (3) in a transversely unbounded region, one seeks a representation for the transverse field vectors $\bar{E}_{t}$ and $\bar{H}_{t}$ in terms of a complete set of vector eigenfunctions. If these eigenfunctions belong to a "characteristic" set for the configuration in question, they are solutions of the sourcefree field equations, satisfy simple orthogonality conditions, and their $z$-dependence in a homo- 
geneous medium is of the form $\exp \left( \pm i \kappa_{n} z\right)$, where $\kappa_{n}$ is the longitudinal wave number and the subscript distinguishes the $n$th mode. Such a set of functions has been found by Arbel and Felsen [1963] and utilized by them for the representation of fields in piecewise homogeneous regions; the resulting "ordinary" and "extraordinary" modes, familiar from conventional magnetoionic theory, are uncoupled in each homogeneous section but couple at the boundaries. If the eigenfunctions turn out to be independent of the properties of the medium in the transverse plane, then they lead to an uncoupled description even in the presence of continuous stratification. This is not the case for the characteristic modes and leads to the coupling difficulties mentioned in the introduction.

By the alternative approach explored in this paper, the mode set is chosen so as to satisfy the source-free field equations when the dielectric tensor is diagonal (uniaxial or isotropic case). In this instance, the resulting eigenfunctions are indeed independent of the transverse medium parameters (they are in fact identical to the transverse $E$ and $H$ mode functions for isotropic regions) and thus lead to an uncoupled representation for arbitrary $\epsilon_{1}(z)$ and $\epsilon_{3}(z)$ when $\epsilon_{2}=0$.

We therefore assume the field representation [Felsen, 1964, sec. 2.2]

$$
\left.\begin{array}{c}
\bar{E}_{t}=\sum_{n} V_{n}^{\prime}(z) \bar{e}_{t n}^{\prime}(\bar{\rho})+\sum_{n} V_{n}^{\prime \prime}(z) \bar{e}_{t n}^{\prime \prime}(\bar{\rho}), \\
\bar{H}_{t}=\sum_{n} I_{n}^{\prime}(z) \bar{h}_{t n}^{\prime}(\bar{\rho})+\sum_{n} I_{n}^{\prime \prime}(z) \bar{h}_{t n}^{\prime \prime}(\bar{\rho}),
\end{array}\right\}
$$

where $\bar{e}_{t n}$ and $\bar{h}_{t n}$ are the transverse $E$ and $H$ mode functions [see (7) and (15)], the $V^{\prime}$ s and $I^{\prime}$ s are voltage and current coefficients (yet to be determined), $\bar{h}_{t n}=\bar{z}_{0} \times \bar{e}_{t n}, \bar{\rho}$ is the transverse position vector, and the single and double primes refer to $E$ modes and $H$ modes, respectively. The summation is over all values of the modal index $n$ (a double index), which is wholly or partially continuous for the present case of an unbounded cross section. For example, if the cross section is described in cylindrical coordinates the eigenfunctions and eigenvalues take the form given in (15) and the first paragraph of section 2.3a.

On inserting (4) in place of the transverse field components in (3), and using the known orthogonality properties of the eigenvectors and the equivalence $\bar{e}_{t n}^{\prime}=\bar{h}_{t n}^{\prime \prime}, \bar{e}_{t n}^{\prime \prime}=-\bar{h}_{t n}^{\prime}$ for modes in an unbounded transverse cross section [Felsen, 1964], one obtains the following two sets of differential equations for the modal amplitudes:

$$
\left.\begin{array}{l}
-\frac{d V_{n}^{\prime}}{d z}=-i \kappa_{n}^{\prime} Z_{c n}^{\prime} I_{n}^{\prime}+v_{n}^{\prime} \\
-\frac{d I_{n}^{\prime}}{d z}=-i \frac{\kappa_{n}^{\prime}}{Z_{c n}^{\prime}} V_{n}^{\prime}+\omega \epsilon_{0} \epsilon_{2} V_{n}^{\prime \prime}+i_{n}^{\prime}
\end{array}\right\}
$$

where $\kappa_{n}$ and $Z_{c n}$ are the modal propagation constant and characteristic impedance, respectively,

$$
\begin{aligned}
& \kappa_{n}^{\prime}=\sqrt{\frac{\epsilon_{1}}{\epsilon_{3}} \sqrt{k^{2} \epsilon_{3}-k_{t n}^{2}}, Z_{c n}^{\prime}=\frac{\kappa_{n}^{\prime}}{\omega \epsilon_{0} \epsilon_{1}},} \\
& \kappa_{n}^{\prime \prime}=\sqrt{k^{2} \epsilon_{1}-k_{t n}^{2}}, Z_{c n}^{\prime \prime}=\frac{\omega \mu_{0}}{\kappa_{n}^{\prime \prime}},
\end{aligned}
$$


and $k_{t n}$ is the transverse eigenvalue corresponding to the modal index $n$. (In a cylindrical coordinate representation, $k_{t n}$ is equal to the radial eigenvalue $k_{t}$.) The quantities $i_{n}^{\prime}, v_{n}^{\prime}, i_{n}^{\prime \prime}$, and $v_{n}^{\prime \prime}$ are the modal voltage and current sources which are obtained from the current densities $\bar{J}$ and $\bar{M}$ as follows:

$$
\left.\begin{array}{l}
i_{n}^{\prime}=\iint_{S} d S \hat{\overline{J_{t}}} \cdot{\overline{e_{t n}^{\prime}}}_{t}, i_{n}^{\prime \prime}=\iint_{S} d S \hat{\bar{J}}_{t} \cdot{\overline{e_{t n}^{\prime \prime}}}^{*}, \\
v_{n}^{\prime}=\iint_{S} d S \hat{\bar{M}_{t}} \cdot \bar{h}_{t n}^{\prime *}, v_{n}^{\prime \prime}=\iint_{S} d \hat{S} \hat{\bar{M}}_{t} \cdot \bar{h}_{t n}^{\prime \prime}, \\
\overline{e_{t n}^{\prime}}=\bar{h}_{t n}^{\prime \prime}=-\frac{\nabla_{t} \phi_{n}}{k_{t n}},{\overline{e_{t n}^{\prime \prime}}}_{t n}=-\bar{h}_{t n}^{\prime}=\bar{h}_{t n}^{\prime \prime} \times \bar{z}_{0},
\end{array}\right\}
$$

where the integrals are taken over that portion of the transverse cross section which is occupied by the sources. $\phi_{n}$ denotes a normalized scalar eigenfunction (see beginning of sec. $2.3 \mathrm{a}$ ).

Equation (5) contains the differential equations for the $E$ mode voltage and current coefficients, whereas (6) is for the $H$ mode quantities. Each set contains a term which cross couples the modes, the coupling coefficient being the same in both cases, namely $\omega \epsilon_{0} \epsilon_{2}$. Consequently, the cross coupling is proportional to the degree of gyrotropy of the medium as represented by $\epsilon_{2}$ and exists whenever this parameter is nonvanishing. When $\epsilon_{2}=0,(5)$ and (6) reduce to the uniaxial forms [Felsen, 1964] and are uncoupled. For the isotropic medium, $\epsilon_{1}=\epsilon_{3}$ and $\epsilon_{2}=0$, in which instance the modes are uncoupled and $\kappa_{n}^{\prime}=\kappa_{n}^{\prime \prime}$, as expected. Since $\epsilon_{1}, \epsilon_{2}$, and $\epsilon_{3}$ are functions of $z,(5)$ and (6) are differential equations with variable coefficients. They may be interpreted in network form, with $V_{n}{ }^{\prime}$ and $I_{n}{ }^{\prime}$ representing the voltage and current on an $E$ mode nonuniform transmission line; $V_{n}^{\prime \prime}$ and $I_{n}{ }^{\prime \prime}$ denote the corresponding $H$ mode quantities. In such a representation the lines are coupled everywhere along their lengths, and are excited by the generators, $i_{n}{ }^{\prime}, v_{n}{ }^{\prime}, i_{n}{ }^{\prime \prime}$, and $v_{n}{ }^{\prime \prime}$.

Since $\epsilon_{2}$ in (2) contains $X$ and $Y$ but not their derivatives, cross coupling in the $E$ and $H$ mode representation does not depend explicitly on the rate of variation of the medium. This result is unlike that obtained from the formulation in terms of ordinary and extraordinary modes in which the rate of variation enters directly into the coupling terms [Fosterling, 1942; Clemmow and Heading, 1954]. Consequently, the $E$ and $H$ mode coupling coefficient is not necessarily large for large gradients, and the utility of the solution need not be restricted to the slowly varying case.

Upon solving (5) and (6) simultaneously for the modal voltages and currents (subject to as yet unspecified boundary conditions) and then placing these quantities into (4) we obtain in principle, the formal solution for the transverse fields. The $z$ components are then derived via [Arbel and Felsen, 1963]:

$$
\left.\begin{array}{l}
E_{z}=-\frac{1}{i \omega \epsilon_{0} \epsilon_{3}} \sum_{n} I_{n}^{\prime} \nabla_{t} \cdot \bar{e}_{t n}^{\prime}+\frac{\mathrm{J}_{z}}{i \omega \epsilon_{0} \epsilon_{3}} \\
H_{z}=-\frac{1}{i \omega \mu_{0}} \sum_{n} V_{n}^{\prime \prime} \nabla_{t} \cdot \bar{e}_{t n}^{\prime}+\frac{M_{z}}{i \omega \mu_{0}}
\end{array}\right\}
$$

Because of the complication introduced by the cross coupling terms in (5) and (6), it is in general quite difficult to provide explicit expressions for the field components in a nonuniform, gyrotropic medium. However, by making $\epsilon_{2}$ small enough, the equations are amenable to treatment by an iterative process, as will be demonstrated. It may also be noted [Gross, 1964] that it is possible to transform (5) and (6) so that the resultant modes are uncoupled and independent when the medium is assumed uniform but gyrotropic. The solution in this case is the same as that found by Arbel and Felsen [1963]. 


\subsection{Iterative Expansion}

From (2), it is seen that $\epsilon_{2}$ will be small if $Y \ll 1$ or $Y \gg 1$. The expansion will be developed for the latter case only, but may be carried out in an analogous fashion for the former case [Gross, 1964].

Assuming that $Y$ is a very large but finite constant (i.e., the inhomogeneity rests solely in the parameter $X$ ), we represent the modal voltage and current coefficients in series of powers of $1 / Y$ as follows (the modal indices $n$ will be omitted for convenience):

$$
V=\sum_{m=0}^{\infty} \frac{V_{m}}{Y^{m}}, \quad I=\sum_{m=0}^{\infty} \frac{I_{m}}{Y^{m}}
$$

and a similar expansion is performed for $\epsilon_{1}$ and $\epsilon_{2}\left(\epsilon_{3}\right.$ is independent of $\left.Y\right)$. On inserting these expansions into (5) and (6) and equating terms with equal powers of $1 / Y$, we obtain a set of differential equations for each iterative order. The zeroth order equations are those for the uniaxial voltage and current coefficients,

$$
\left.\begin{array}{l}
\frac{d V_{0}^{\prime}}{d z}-i \frac{k^{2}(1-X)-k_{t}^{2}}{\omega \epsilon_{0}(1-X)} I_{0}^{\prime}=v^{\prime}, \quad \frac{d I_{0}^{\prime}}{d z}-i \omega \epsilon_{0} V_{0}^{\prime}=i^{\prime}, \\
\frac{d V_{0}^{\prime \prime}}{d z}-i \omega \mu_{0} I_{0}^{\prime \prime}=v^{\prime \prime}, \frac{d I_{0}^{\prime \prime}}{d z}-\frac{i}{\omega \mu_{0}}\left(k^{2}-k_{t}^{2}\right) V_{0}^{\prime \prime}=i^{\prime \prime},
\end{array}\right\}
$$

whereas, the $m$ th order gyrotropic corrections to the uniaxial coefficients are specified by (note: quantities with negative index vanish identically),

$$
\left.\begin{array}{l}
\frac{d V_{m}^{\prime}}{d z}-i \frac{k^{2}(1-X)-k_{t}^{2}}{\omega \epsilon_{0}(1-X)} I_{m}^{\prime}=0, \\
\frac{d I_{m}^{\prime}}{d z}-i \omega \epsilon_{0} V_{m}^{\prime}=-\omega \epsilon_{0} X\left[V_{m-1}^{\prime \prime}+V_{m-3}^{\prime \prime}+\cdots-i\left(V_{m-2}^{\prime}+V_{m-4}^{\prime}+\cdots\right)\right] \\
\frac{d V_{m}^{\prime \prime}}{d z}-i \omega \mu_{0} I_{m}^{\prime \prime}=0 \\
\frac{d I_{m}^{\prime \prime}}{d z}-\frac{i}{\omega \mu_{0}}\left(k^{2}-k_{t}^{2}\right) V_{m}^{\prime \prime}=\omega \epsilon_{0} X\left[V_{m-1}^{\prime}+V_{m-3}^{\prime}+\cdots+i\left(V_{m-2}^{\prime \prime}+V_{m-4}^{\prime \prime}+\cdots\right)\right] .
\end{array}\right\}
$$

It is seen that the true sources appear only in the zeroth order solution and that the source for the $m$ th order coefficients is provided by the voltages of lower order. Since the homogeneous forms of (10) and (11) are identical, the solutions for any order may be derived from the same Green's function, and the $E$ and $H$ mode equations are no longer coupled. The complete solution may therefore be obtained, in principle, by an iterative procedure once the uniaxial result has been determined. It may be mentioned that the $H$ mode coefficients are independent of the medium, in consequence of the perturbation about the uniaxial case which does not influence the $H$ mode fields [Felsen, 1964].

\subsection{Excitation by a Transverse Electric Current Element}

\section{a. Voltage and Current Coefficients}

To illustrate the form of the solution obtained from the preceding analysis, we consider the case of a transverse electric current element located at $z=z^{\prime}$ on the $z$-axis and oriented parallel to the $x$-axis. Cylindrical coordinates $(\rho, \psi, z)$ will be used, where $\rho$ is the radial distance from the $z$-axis in the transverse plane, and $\psi$ is the angle between the radius vector $\bar{\rho}$ and the $x$-axis. 
In this coordinate system the modal index $n$ is described by the two quantities $k_{t}$ and $\nu$ which represents the eigenvalues in the angular and radial domains, respectively, and the normalized scalar mode function is given by

$$
\phi_{n}=\left(k_{t} / 2 \pi\right)^{1 / 2} \exp (i \nu \psi) J_{\nu}\left(k_{t} \rho\right), \nu=0, \pm 1, \pm 2 \ldots, 0<k_{t}<\infty
$$

(Felsen, 1964, part 2). For a tranverse current source, located as mentioned above, the only nonvanishing modes are those for which $\nu= \pm 1$. Furthermore, a transverse electric current element gives rise to $v_{n}^{\prime}=v_{n}^{\prime \prime}=0$ for all modes, as may be seen from (7). Then with $\bar{J}=\bar{x}_{0} \delta\left(z-z^{\prime}\right) \delta(\bar{\rho})$, where $\bar{x}_{0}$ is a unit vector in the $x$-direction and $\delta(\alpha)$ is the delta function, we find from (7) (see also (15)):

$$
\left.\begin{array}{c}
i_{n}^{\prime} \equiv a_{n}^{\prime} \delta\left(z-z^{\prime}\right)=\mp \frac{1}{2} \sqrt{\frac{k_{t}}{2 \pi}} \delta\left(z-z^{\prime}\right) \\
i_{n}^{\prime \prime} \equiv a_{n}^{\prime \prime} \delta\left(z-z^{\prime}\right)=\frac{i}{2} \sqrt{\frac{k_{t}}{2 \pi}} \delta\left(z-z^{\prime}\right)
\end{array}\right\}
$$

where it is understood that $n$ now signifies the double subscript $\left(k_{t}, \pm 1\right) . \quad a_{n}^{\prime}$ and $a_{n}^{\prime \prime}$ denote the strengths of the current generators located on the transmission lines at $z^{\prime}$.

For the zero order $E$ mode coefficients $\left[V_{m n}^{\prime}\right.$ and $I_{m n}^{\prime}$, with $m=0$ in (9)],

$$
\begin{gathered}
V_{0 n}^{\prime}\left(z, z^{\prime}\right)=-a_{n}^{\prime} Z_{k t}^{\prime}\left(z, z^{\prime}\right) \\
I_{0 n}^{\prime}\left(z, z^{\prime}\right)=-a_{n}^{\prime} T_{k t}^{I^{\prime}}\left(z, z^{\prime}\right)
\end{gathered}
$$

where $Z_{k t}^{\prime}\left(z, z^{\prime}\right)$ and $T_{k t}^{I^{\prime}}\left(z, z^{\prime}\right)$ are voltage and current Green's functions [Felsen, 1964, sec. 2.3] for the uncoupled $E$ mode uniaxial transmission lines excited by a current generator of unit strength at $z^{\prime}$. The same expressions apply for $H$ modes, with double primes replacing the single primes. Since the Green's functions depend on $k_{t}$ but not on $\nu$ [see (22) and (23), with $\kappa, Y$, and $Z$ given in (6)], the latter index has been omitted in the suffix. In the first order equations, the zero order voltage coefficients $V_{0 n}^{\prime}$ and $V_{0 n}^{\prime \prime}$ act as continuously distributed current generators, as may be seen from (11). Thus, the total voltage and current coefficients for the modes $n=\left(k_{t}, \pm 1\right)$ are to $0(1 / Y)$,

$$
\begin{aligned}
& V_{n}^{\prime}= \pm \frac{1}{2} \sqrt{\frac{k_{t}}{2 \pi}} Z_{k t}^{\prime}\left(z, z^{\prime}\right)-\frac{i}{2} \sqrt{\frac{k_{t}}{2 \pi}} \frac{\omega \epsilon_{0}}{Y} \int_{-\infty}^{\infty} X\left(z^{\prime \prime}\right) Z_{k t}^{\prime}\left(z, z^{\prime \prime}\right) Z_{k t}^{\prime \prime}\left(z^{\prime \prime}, z^{\prime}\right) d z^{\prime \prime}+0\left(1 / Y^{2}\right) \\
& I_{n}^{\prime}= \pm \frac{1}{2} \sqrt{\frac{k_{t}}{2 \pi}} T_{k t}^{I^{\prime}}\left(z, z^{\prime}\right)-\frac{i}{2} \sqrt{\frac{k_{t}}{2 \pi}} \frac{\omega \epsilon_{0}}{Y} \int_{-\infty}^{\infty} X\left(z^{\prime \prime}\right) T_{k t}^{I^{\prime}}\left(z, z^{\prime \prime}\right) Z_{k t}^{\prime \prime}\left(z^{\prime \prime}, z^{\prime}\right) d z^{\prime \prime}+0\left(1 / Y^{2}\right) \\
& V_{n}^{\prime \prime}=-\frac{i}{2} \sqrt{\frac{k_{t}}{2 \pi}} Z_{k t}^{\prime \prime}\left(z, z^{\prime}\right) \mp \frac{1}{2} \sqrt{\frac{k_{t}}{2 \pi}} \frac{\omega \epsilon_{0}}{Y} \int_{-\infty}^{\infty} X\left(z^{\prime \prime}\right) Z_{k t}^{\prime \prime}\left(z, z^{\prime \prime}\right) Z_{k t}^{\prime}\left(z^{\prime \prime}, z^{\prime}\right) d z^{\prime \prime}+0\left(1 / Y^{2}\right) \\
& I_{n}^{\prime \prime}=-\frac{i}{2} \sqrt{\frac{k_{t}}{2 \pi}} T_{k t}^{l^{\prime \prime}}\left(z, z^{\prime}\right) \mp \frac{1}{2} \sqrt{\frac{k_{t}}{2 \pi}} \frac{\omega \epsilon_{0}}{Y} \int_{-\infty}^{\infty} X\left(z^{\prime \prime}\right) T_{k t}^{\prime \prime}\left(z, z^{\prime \prime}\right) Z_{k t}^{\prime}\left(z^{\prime \prime}, z^{\prime}\right) d z^{\prime \prime}+0\left(1 / Y^{2}\right) .
\end{aligned}
$$

Where multiple signs occur, the upper sign goes with $\nu=+1$, and the lower with $\nu=-1$. One observes that the transverse electric source excites gyrotropic corrections of the order $1 / Y$ in both the $E$ and $H$ mode coefficients. This behavior occurs also with a transverse magnetic current source, whereas for longitudinal sources the gyrotropic effects are of higher order [Gross, 1964]. 
For a longitudinal electric current element the first $E$ mode gyrotropic correction is in the $1 / Y^{2}$ term, while the total $H$ mode coefficients are of $0(1 / Y)$.

For the nearly isotropic medium $(Y<<1)$, similar results can be derived readily.

\section{b. Field Components}

In a cylindrical geometry the vector eigenfunctions are given by

$$
\left.\begin{array}{l}
{\overline{e_{k t, \nu}^{\prime}}}^{\prime}(\rho, \psi)=\bar{h}_{k t, \nu}^{\prime \prime}(\rho, \psi)=-\sqrt{\frac{k_{t}}{2 \pi}} e^{i \nu \psi}\left\{\bar{\rho}_{0} \frac{1}{2}\left(J_{\nu-1}\left(k_{t} \rho\right)-J_{\nu+1}\left(k_{t} \rho\right)\right)+\bar{\psi}_{0} \frac{i \nu}{k_{t} \rho} J_{v}\left(k_{t} \rho\right)\right\} \\
{\overline{e_{k t, \nu}^{\prime \prime}}}^{\prime \prime}(\rho, \psi)=-\bar{h}_{k t, \nu}^{\prime}(\rho, \psi)=\overline{e_{k t, \nu}^{\prime}}(\rho, \psi) \times z_{0}
\end{array}\right\}
$$

where $J_{\nu}$ is the Bessel function, and $\bar{\rho}_{0}$ and $\bar{\psi}_{0}$ are unit vectors in the $\rho$ and $\psi$ directions, respectively. The field components are obtained from (4), (8), and (14), with $\sum_{n} \rightarrow \sum_{\nu=-\infty}^{\infty} \int_{0}^{\infty} d k_{t} k_{t}$; only the $\nu= \pm]$ terms contribute when the $z$-axis passes through the source. The following expressions result for the field components in a cylindrical coordinate representation:

$E_{\rho_{\psi}}=\frac{1}{4 \pi} \int_{0}^{\infty} d k_{t} k_{t}\left\{\begin{array}{l}\cos \psi \\ \sin \psi\end{array}\left[\mp J_{0}\left(\mathrm{k}_{t} \rho\right)\left(Z_{k t}^{\prime}\left(z, z^{\prime}\right)+Z_{k t}^{\prime \prime}\left(z, z^{\prime}\right)\right)+J_{2}\left(k_{t} \rho\right)\left(Z_{k t}^{\prime}\left(z, z^{\prime}\right)-Z_{k t}^{\prime \prime}\left(z, z^{\prime}\right)\right)\right]\right.$

$$
\begin{aligned}
& -\frac{\omega \epsilon_{0}}{Y} \sin \psi\left[J_{0}\left(k_{t} \rho\right) \int_{-\infty}^{\infty} d z^{\prime \prime} X\left(z^{\prime \prime}\right)\left(Z_{k t}^{\prime}\left(z, z^{\prime \prime}\right) Z_{k t}^{\prime \prime}\left(z^{\prime \prime}, z^{\prime}\right)+Z_{k t}^{\prime \prime}\left(z, z^{\prime \prime}\right) Z_{k t}^{\prime}\left(z^{\prime \prime}, z^{\prime}\right)\right)\right. \\
& \left.\left.\quad \mp J_{2}\left(k_{t} \rho\right) \int_{-\infty}^{\infty} d z^{\prime \prime} X\left(z^{\prime \prime}\right)\left(Z_{k t}^{\prime}\left(z, z^{\prime \prime}\right) Z_{k t}^{\prime \prime}\left(z^{\prime \prime}, z^{\prime}\right)-Z_{k t}^{\prime \prime}\left(z, z^{\prime \prime}\right) Z_{k t}^{\prime}\left(z^{\prime \prime}, z^{\prime}\right)\right)\right]\right\}+0\left(1 / Y^{2}\right)
\end{aligned}
$$

where the upper algebraic signs and trigonometric functions refer to $E_{\rho}$, and the lower symbols are appropriate to $E_{\psi}$.

Also,

$$
E_{z}=\frac{i}{2 \pi \omega \epsilon_{0} \epsilon_{3}} \int_{0}^{\infty} d k_{t} k_{t}^{2} J_{1}\left(k_{t} \rho\right)\left\{T_{k t}^{I^{\prime}}\left(z, z^{\prime}\right) \cos \psi+\frac{\omega \epsilon_{0}}{Y} \int_{-\infty}^{\infty} d z^{\prime \prime} X\left(z^{\prime \prime}\right) T_{k t}^{\prime \prime}\left(z, z^{\prime \prime}\right) Z_{k t}^{\prime \prime}\left(z^{\prime \prime}, z^{\prime}\right)\right\}+0\left(1 / Y^{2}\right) .
$$

Similarly,

$$
\begin{gathered}
H_{\rho_{\psi}}=-\frac{1}{4 \pi} \int_{0}^{\infty} d k_{t} k_{t}\left\{\begin{array}{c}
\sin \psi \\
\cos \psi
\end{array}\left[J_{0}\left(k_{t} \rho\right)\left(T_{k t}^{I^{\prime}}\left(z, z^{\prime}\right)+T_{k t}^{I^{\prime \prime}}\left(z, z^{\prime}\right)\right) \pm J_{2}\left(k_{t} \rho\right)\left(T_{k t}^{I^{\prime}}\left(z, z^{\prime}\right)-T_{k t}^{l^{\prime \prime}}\left(z, z^{\prime}\right)\right)\right]\right. \\
\mp \frac{\omega \epsilon_{0}}{Y} \begin{array}{c}
\cos \psi \\
\sin \psi
\end{array}\left[J_{0}\left(k_{t} \rho\right) \int_{-\infty}^{\infty} d z^{\prime \prime} X\left(z^{\prime \prime}\right)\left(T_{k t}^{I^{\prime}}\left(z, z^{\prime \prime}\right) Z_{k t}^{\prime \prime}\left(z^{\prime \prime}, z^{\prime}\right)+T_{k t}^{I^{\prime \prime}}\left(z, z^{\prime \prime}\right) Z_{k t}^{\prime}\left(z^{\prime \prime}, z^{\prime}\right)\right)\right. \\
\left. \pm J_{2}\left(k_{t} \rho\right) \int_{-\infty}^{\infty} d z^{\prime \prime} X\left(z^{\prime \prime}\right)\left(T_{k t}^{I^{\prime}}\left(z, z^{\prime \prime}\right) Z_{k t}^{\prime \prime}\left(z^{\prime \prime}, z^{\prime}\right)-T_{k t}^{I^{\prime \prime}}\left(z, z^{\prime \prime}\right) Z_{k t}^{\prime}\left(z^{\prime \prime}, z^{\prime}\right)\right]\right\}+0\left(1 / Y^{2}\right)
\end{gathered}
$$

Finally,

$$
H_{z}=\frac{i}{2 \pi \omega \mu_{0}} \int_{0}^{\infty} d k_{t} k_{t}^{2} J_{1}\left(k_{t} \rho\right)\left\{Z_{k t}^{\prime \prime}\left(z, z^{\prime}\right)-\frac{\omega \epsilon_{0}}{Y} \int_{-\infty}^{\infty} d z^{\prime \prime} X\left(z^{\prime \prime}\right) Z_{k t}^{\prime \prime}\left(z, z^{\prime \prime}\right) Z_{k_{t}}^{\prime}\left(z^{\prime \prime}, z^{\prime}\right)\right\}+0\left(1 / Y^{2}\right) .
$$

The zero order expressions (in $1 / Y$ ) in these equations yield the uniaxial field components in terms of uncoupled $E$ and $H$ modes, whereas the first order terms give the first gyrotropic corrections. The solutions for the field components in (16) to (19) must still be regarded as formal since 
it is necessary to substitute for the Green's functions and carry out the integration. This will be demonstrated below in a simple example. More complex problems have also been examined [Gross, 1964] and will be submitted separately.

Since the iterative solution involves a power series in $1 / Y$, there may be some question concerning its convergence. In view of the complicated nature of the coefficients in the series, a general statement is difficult and we must trust the expectation that reliable results may be obtained with a few terms when $Y$ is sufficiently large. The following observation is helpful in this connection: since each integral in the iterated solution contains the factor $X(z)$ (the $m$ th order term involves an $m$-fold integration), the $m$ th order term may behave according to $\left(X_{m} / Y\right)^{m}$ where $X_{m}$ denotes the maximum value of $X(z)$ in the integration interval. Thus, it is to be anticipated that the expansion is useful when $\left(X_{m} / Y\right)<<1$. The restriction $\left(X_{m} / Y\right)<1$ is well motivated since this range, but not the complementary one, may be regarded as a perturbation about the uniaxial case. In certain configurations, such as the semi-infinite medium with monotonically increasing electron density, $X$ may reach values in excess of $Y$ for distant regions along the $z$-axis. If slight losses are included in these cases, it can be shown [Gross, 1964] that these regions will not limit the convergence. Instead the rate of variation of the medium parameters over a free space wavelength must be made small compared to $Y$; this condition appears in (44) as $\lambda<<2 \pi Y$.

\subsection{Construction of the Modal Green's Functions}

As noted in sections 2.2 and 2.3, the iterative solution may be built up entirely from the Green's functions for the transmission line equations (10) or (11), the functions being identical since these equations differ only in their source terms. The various Green's functions have a direct physical interpretation: $Y_{n}\left(z, z^{\prime}\right)$ and $T_{n}{ }^{V}\left(z, z^{\prime}\right)$ denote the current and voltage, respectively, observed at $z$ due to excitation by a unit strength voltage point source at $z^{\prime}$, while $Z_{n}\left(z, z^{\prime}\right)$ and $T_{n}{ }^{I}\left(z, z^{\prime}\right)$ denote the voltage and current, respectively, observed at $z$ due to excitation by a unit strength current source at $z^{\prime}$. While the transverse electric dipole element discussed above does not give rise to a voltage source, thereby requiring the use of $Z_{n}\left(z, z^{\prime}\right)$ and $T_{n}{ }^{I}\left(z, z^{\prime}\right)$ only, all of the Green's functions are needed for a description of the field with more general excitation. Since the voltage and current at a source-free point are connected by the transmission line equations,

$$
\frac{d V_{n}\left(z, z^{\prime}\right)}{d z}=i \kappa_{n} Z_{c n} I_{n}\left(z, z^{\prime}\right), \quad \frac{d I_{n}\left(z, z^{\prime}\right)}{d z}=i \kappa_{n} Y_{c n} V_{n}\left(z, z^{\prime}\right)
$$

and in view of the reciprocity relations [Felsen and Marcuvitz, 1956, 1959]

$$
T_{n}^{I}\left(z, z^{\prime}\right)=-T_{n}^{V}\left(z^{\prime}, z\right), \quad Y_{n}\left(z, z^{\prime}\right)=Y_{n}\left(z^{\prime}, z\right), \quad Z_{n}\left(z, z^{\prime}\right)=Z_{n}\left(z^{\prime}, z\right)
$$

all of the Green's functions may be derived from the knowledge of any particular one. In (20), $\kappa_{n}, Z_{c n}$, and $Y_{c n}=1 / Z_{c n}$ represent the previously defined propagation constant, characteristic impedance and characteristic admittance, respectively, all of which may be $z$-dependent (the identification of these quantities in (10) or (11) is evident). It is usually convenient to determine either $Y_{n}\left(z, z^{\prime}\right)$ or $Z_{n}\left(z, z^{\prime}\right)$, with the former and latter preferable for the $E$ and $H$ mode problems, respectively. In terms of $Y_{n}\left(z, z^{\prime}\right)$,

$$
\begin{aligned}
& T_{n}^{V}\left(z, z^{\prime}\right)=\frac{1}{i \kappa_{n}(z) Y_{c n}(z)} \frac{d}{d z} Y_{n}\left(z, z^{\prime}\right), \quad T_{n}^{I}\left(z, z^{\prime}\right)=-\frac{1}{i \kappa_{n}\left(z^{\prime}\right) Y_{c n}\left(Z^{\prime}\right)} \frac{d}{d z^{\prime}} Y_{n}\left(z, z^{\prime}\right) \\
& Z_{n}\left(z, z^{\prime}\right)=\frac{1}{\kappa_{n}(z) Y_{c n}(z) \kappa_{n}\left(z^{\prime}\right) Y_{c n}\left(z^{\prime}\right)} \frac{d^{2}}{d z d z^{\prime}} Y_{n}\left(z, z^{\prime}\right)
\end{aligned}
$$

with dual relations applicable when $Z_{n}\left(z, z^{\prime}\right)$ is given at the outset. 


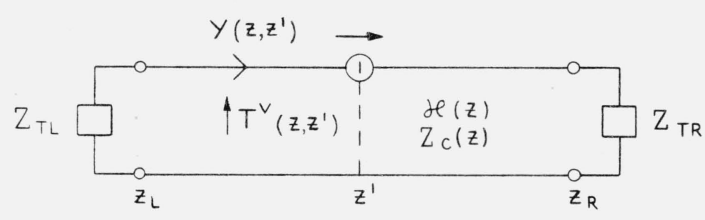

FIGURE 1. Nonuniform section of transmission line.

The general network problem for the determination of $Y\left(z, z^{\prime}\right)$ is shown in figure 1 (the modal subscript $n$ is omitted henceforth); in mathematical terms, $Y\left(z, z^{\prime}\right)$ satisfies the second-order differential equation obtained after elimination of $T^{v}$ from the transmission line equations:

$$
\left[\frac{d}{d z} \frac{1}{\kappa(z) Y_{c}(z)} \frac{d}{d z}+\kappa(z) Z_{c}(z)\right] Y\left(z, z^{\prime}\right)=i \delta\left(z-z^{\prime}\right) .
$$

The nonuniform transmission line described by the parameters $\kappa(z), Z_{c}(z)$ is assumed to be terminated at $z_{L}$ and $z_{R}$ by impedances $Z_{T L}$ and $Z_{T R}$, respectively, which prescription specifies the boundary conditions at the $z$-termini of the region covered by (23). ${ }^{2}$ The construction of the Green's function is conventional: we assume that the two linearly independent solutions of the homogeneous equation (23) are superposed in such a manner as to yield two (current) functions $I_{L}(z)$ and $I_{R}(z)$ which satisfy the boundary conditions at $z_{L}$ and $z_{R}$, respectively. Then

$$
Y\left(z, z^{\prime}\right)=\frac{I_{L}\left(z_{<}\right) I_{R}\left(z_{>}\right)}{\frac{Z_{c}(z)}{i \kappa(z)} \Delta\left(I_{L}, I_{R}\right)}, \Delta\left(I_{L}, I_{R}\right)=I_{L} \frac{d I_{R}}{d z}-I_{R} \frac{d I_{L}}{d z},
$$

where $z_{<}$equals $z$ when $z<z^{\prime}$ and $z^{\prime}$ when $z>z^{\prime}$, with the converse applying to $z_{>}$. The denominator in (24) (the Wronskian) is a constant. Upon introducing the impedances,

$$
\overleftarrow{Z}(z)=-\frac{V_{L}(z)}{I_{L}(z)}=-\frac{d I_{L}(z) / d z}{i \kappa(z) Y_{c}(z) I_{L}(z)}, \quad \vec{Z}(z)=\frac{d I_{R}(z) / d z}{i \kappa(z) Y_{c}(z) I_{R}(z)}
$$

seen to the left and right, respectively, of the point $z$, one may write (24) in the normalized form,

$$
Y\left(z, z^{\prime}\right)=\frac{\left[I_{L}\left(z_{<}\right) / I_{L}\left(z^{\prime}\right)\right]\left[I_{R}\left(z_{>}\right) / I_{R}\left(z^{\prime}\right)\right]}{\overleftrightarrow{Z}\left(z^{\prime}\right)},
$$

where

$$
\overleftrightarrow{Z}\left(z^{\prime}\right)=\overleftarrow{Z}\left(z^{\prime}\right)+\vec{Z}\left(z^{\prime}\right)
$$

The detailed structure of $I_{L}$ and $I_{R}$ may be exhibited either in a standing wave [Felsen and Marcuvitz, 1959] or a traveling wave representation. For the nonuniform transmission line problem in question, the latter is frequently more convenient and provides an insight into the physical mechanism of reflection and refraction in a progressing wave. We shall therefore break the total currents into two parts

$$
I_{L}=I_{L}^{+}+I_{L}^{-}, I_{R}=I_{R}^{+}+I_{R}^{-}
$$

${ }^{2}$ Evidently, no mode coupling is assumed to take place at the boundary. This obtains when the regions $z<z_{L}$ and $z>z_{R}$ are occupied by an isotropic or by a different anisotropic medium (with optic axis along $z$ ), as long as the analysis is performed by the $E$ and $H$ mode decomposition. 
with superscripts + and - denoting waves traveling in the $(+z)$ and $(-z)$ directions, respectively. While these distinctions become obscured in an inhomogeneous region where continuous reflections take place, they may be made precise by the requirement that the + wave satisfy the boundary conditions at $z \rightarrow \infty$ (radiation condition) if the medium is infinite in extent, with an analogous requirement imposed on the - wave at $z \rightarrow-\infty$. Thus,

$$
\overleftarrow{Z_{i}}(z)=-\frac{d I^{-}(z) / d z}{i \kappa Y_{c} I^{-}(z)}, \quad \vec{Z}_{i}(z)=\frac{d I^{+}(z) / d z}{i \kappa Y_{c} I^{+}(z)}
$$

represent the input impedances to the unbounded medium (matched case) seen to the left and right of $z$, respectively, and

$$
\overleftarrow{\Gamma}(z)=\frac{I_{L}^{+}(z)}{I_{L}^{-}(z)}=\frac{\overleftarrow{Z_{i}}(z)-\overleftarrow{Z}(z)}{\vec{Z}_{i}(z)+\overleftarrow{Z}(z)}, \quad \overrightarrow{\Gamma(z)}=\frac{I_{R}^{-}(z)}{I_{R}^{+}(z)}=\frac{\vec{Z}_{i}(z)-\vec{Z}(z)}{\overleftarrow{Z_{i}}(z)+\vec{Z}(z)}
$$

are the (current) reflection coefficients. The reflection coefficients at any two points may be related by the identity

$$
\overleftarrow{\Gamma}(z)=\overleftarrow{\Gamma}\left(z^{\prime}\right) \frac{I_{L}^{-}\left(z^{\prime}\right)}{I_{L}^{+}\left(z^{\prime}\right)} \frac{I_{L}^{+}(z)}{I_{L}^{-}(z)}
$$

with a similar expression for $\vec{\Gamma}(z)$, and it is then not difficult to show that

$$
Y\left(z, z^{\prime}\right)=\frac{\left[\hat{I}_{L}^{-}\left(z_{<}\right)+\overleftarrow{\Gamma}\left(z_{L}\right) \hat{I}_{L}^{+}\left(z_{<}\right)\right]\left[\hat{I}_{R}^{+}\left(z_{>}\right)+\vec{\Gamma}\left(z_{R}\right) \hat{I}_{R}^{-}\left(z_{>}\right)\right]}{\overleftrightarrow{Z}_{i}\left(z^{\prime}\right)\left[I_{R}^{+}\left(z^{\prime}\right) \hat{I}_{L}^{-}\left(z^{\prime}\right)-\vec{\Gamma}\left(z_{R}\right) \overleftarrow{\Gamma}\left(z_{L}\right) \hat{I}_{R}^{-}\left(z^{\prime}\right) \hat{I}_{L}^{+}\left(z^{\prime}\right)\right]}
$$

where $\overleftrightarrow{Z_{i}}=\overleftarrow{Z_{i}}+\vec{Z}_{i}$, and

$$
\hat{I_{L}^{ \pm}}(z)=\frac{I_{L}^{ \pm}(z)}{I_{L}^{ \pm}\left(z_{L}\right)}, \quad \hat{I_{R}^{ \pm}}(z)=\frac{I_{R}^{ \pm}(z)}{I_{R}^{ \pm}\left(z_{R}\right)} .
$$

In (31), the reflection coefficients are conveniently those at the endpoints (note: $\vec{Z}\left(z_{R}\right)=Z_{T R}$, $\left.\overleftarrow{Z}\left(z_{L}\right)=Z_{T L}\right)$, and the entire expression may be written out explicitly in terms of the traveling wave solutions of the homogeneous equation (23). It is noted in this connection that one may put $I_{L}^{-}=I_{R}^{-}, I_{L}^{+}=I_{R}^{+}$, since only normalized quantities appear in the formula. An analogous result for the Green's function $Z\left(z, z^{\prime}\right)$ follows from duality considerations.

Equation (31) simplifies considerably for certain special cases. In an infinitely extended region, $\overleftarrow{\Gamma}\left(z_{L}\right)=\vec{\Gamma}\left(z_{R}\right)=0$, and one finds

$$
Y\left(z, z^{\prime}\right)=\frac{\left[I^{-}\left(z_{<}\right) / I^{-}\left(z^{\prime}\right)\right]\left[I^{+}\left(z_{>}\right) / I^{+}\left(z^{\prime}\right)\right]}{\overleftrightarrow{Z}_{i}\left(z^{\prime}\right)}
$$

which formula is also deduced directly from (26). In a semi-infinite region (with $z_{L} \rightarrow-\infty$, i.e. $\left.\overleftarrow{\Gamma}\left(z_{L}\right)=0\right)$

$$
Y\left(z, z^{\prime}\right)=\frac{\left.I^{-}\left(z_{<}\right)\left[\hat{I}_{R}^{+}\left(z_{>}\right)+\vec{\Gamma}^{\hat{\imath}} \hat{a}_{>}\right)\right]}{\overleftrightarrow{Z}_{i}\left(z^{\prime}\right) I^{-}\left(z^{\prime}\right) \hat{I}_{R}^{+}\left(z^{\prime}\right)}
$$


Returning now to the problem at hand after these general considerations, we see from (10) or (11) that the homogeneous equations for $Y \gg 1$ are

$$
\left\{\frac{d^{2}}{d z^{2}}+k^{2}\left[1-\frac{k_{t}^{2}}{k^{2}(1-X)}\right]\right\} I^{\prime}=0 \quad \text { for the } E \text { modes, }
$$

and

$$
\left\{\frac{d^{2}}{d z^{2}}+k^{2}\left[1-\frac{k_{t}^{2}}{k^{2}}\right]\right\} V^{\prime \prime}=0 \quad \text { for the } H \text { modes, }
$$

which results follow also from (23) and its dual, in view of the definitions of $\kappa, Z_{c}$ in (6). The traveling wave solutions for the $H$ modes evidently comprise the exponentials exp $\left[ \pm i \sqrt{k^{2}-k_{t}{ }^{2} z}\right.$, independent of the medium constants, whereas their form for the $E$ modes depend on the details of the stratification $X(z)$.

\section{Application to a Semi-Infinite Medium With Linearly Varying Electron Density}

A simple illustration of the preceding results is now given for the case of a semi-infinite, nearly uniaxial medium with a linear variation of $X$ with $z$ as shown in figure 2. In a subsequent paper, it is planned to cover further details of the derivation, together with applications to various other nonuniform configurations.

The source is taken as the transverse electric current element located at $z=z^{\prime}$ in the vacuum half space $z<z_{b}=-\frac{1}{2}$ (see sec. 2.3). The independent functions $I^{+}$and $I^{-}$for both $E$ and $H$ modes are simple exponentials in the vacuum region (the pertinent equation is the same as (34b) since $X=0)$. In the linear half space the $H$ mode functions remain unaltered; however, the $E$ mode differential equation is solved in terms of Whittaker functions. Throughout the entire space, the $H$ mode Green's function is

$$
Z^{\prime \prime}\left(z, z^{\prime}\right)=\frac{\omega \mu_{0}}{2 \sqrt{k^{2}-k_{t}^{2}}} e^{i \sqrt{k^{2}-k_{t}^{2}}\left|z-z^{\prime}\right|},
$$

a result obtained at once from the dual of (32), with $\overleftrightarrow{Y}_{i}^{\prime \prime}\left(z^{\prime}\right)=2 Y_{c}^{\prime \prime}=2 \kappa^{\prime \prime} / \omega \mu_{0} \quad$ [see (6)]

The $E$ mode Green's function, constructed from (33) (with $z_{R} \equiv z_{b}$ ), may be shown to be given by

$Y\left(z, z^{\prime}\right)=\frac{\omega \epsilon_{0}}{2 \sqrt{k^{2}}-k_{t}^{2}}\left[e^{i \sqrt{k^{2}-k_{t}^{2}} z-z^{\prime} \mid}+\frac{i \sqrt{k^{2}-k_{t}^{2}} W_{1}\left(z_{b}\right)-W_{1}^{\prime}\left(z_{b}\right)}{i \sqrt{k^{2}-k_{t}^{2}} W_{1}\left(z_{b}\right)+W_{1}^{\prime}\left(z_{b}\right)} e^{-i \sqrt{k^{2}-k_{t}^{2}}\left(z+z^{\prime}-2 z_{b}\right)}\right]$

for $z<z_{b}$, and by

$$
Y\left(z, z^{\prime}\right)=i \omega \epsilon_{0} e^{-i \sqrt{k^{2}-k^{2}}\left(z^{\prime}-z_{b}\right)} \frac{W_{1}(z)}{i \sqrt{k^{2}-k_{t}^{2}} W_{1}\left(z_{b}\right)+W_{1}^{\prime}\left(z_{b}\right)}
$$

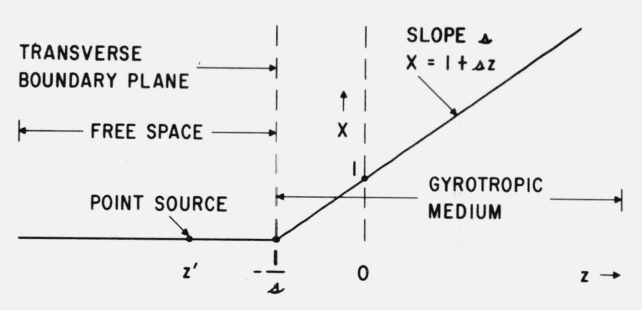

Figure 2. Semi-infinite linear model. 
for $z>z_{b}$, where $z_{b}$ locates the boundary plane separating the two semi-infinite regions shown in figure 2. $W_{1}$ and $W_{2}$ are linearly independent Whittaker functions defined by [Slater, 1960]

$$
\left.\begin{array}{l}
W_{1}(z)=W_{\frac{i k_{t}^{2}}{2 a k}, \frac{1}{2}}(-i 2 k z) \\
W_{2}(z)=W_{\frac{-i k_{t}^{2}}{2 a k}, \frac{1}{2}}(i 2 k z)
\end{array}\right\}
$$

where $s$ is the derivative of $X$ with respect to $z$ and the primes on $W_{1}$ in $(36 \mathrm{a}, \mathrm{b})$ denote $\frac{d}{d z_{b}}$. The reflected wave in (36a) appears to originate from the image point at $2 z_{b}-z^{\prime}$, and the factor multiplying the second exponential is the reflection coefficient $\vec{\Gamma}\left(z_{b}\right)$, which was derived from a knowledge of $\vec{Z}\left(z_{b}\right)$; i.e., of the voltage and current solutions in the plasma half-space. Since $W_{1}(z)$ may be shown to satisfy the radiation condition at $z \rightarrow \infty$, and with $\vec{Z}_{i}\left(z_{b}\right)=\sqrt{k^{2}-k_{t}^{2}} / \omega \epsilon_{0}$ in vacuum, the form of $\vec{\Gamma}\left(z_{b}\right)$ in (36a) follows. The formula in (36b) is obtained at once from the continuity of $Y\left(z, z^{\prime}\right)$ at $z_{b}$ and the above-mentioned behavior of $W_{1}(z)$ at $z \rightarrow \infty$.

The uniaxial portion of the far field solution in the vacuum half-space, obtained from an asymptotic evaluation, is found to be given by [Gross, 1964].

$$
\begin{aligned}
& E_{\theta u}=-\frac{i \omega \mu_{0}}{4 \pi} \cos \theta \cos \psi\left(1-\Gamma^{\prime}\right) \frac{e^{i k r}}{r} \\
& E_{\psi u}=-\frac{i \omega \mu_{0}}{4 \pi} \sin \psi \frac{e^{i k r}}{r} \\
& H_{\theta u}=\frac{i k}{4 \pi} \sin \psi \frac{e^{i k r}}{r} \\
& H_{\psi u}=\frac{-i k}{4 \pi} \cos \theta \cos \psi\left(1-\Gamma^{\prime}\right) \frac{e^{i k r}}{r} \\
& E_{r u}=H_{r u}=0
\end{aligned}
$$

where $(r, \theta, \psi)$ are the spherical coordinates of the observation point relative to the source; $E_{\theta u}$, $E_{\psi u}$, etc., are the spherical field components, and $\Gamma^{\prime}$ is the reflection coefficient

$$
\Gamma^{\prime}=\frac{i k \cos \theta W_{1}\left(z_{b}\right)-W_{1}^{\prime}\left(z_{b}\right)}{i k \cos \theta W_{1}\left(z_{b}\right)+W_{1}^{\prime}\left(z_{b}\right)} e^{-i 2 k\left(z^{\prime}-z_{b}\right) \cos \theta}
$$

The angle $\theta$ here is measured from the negative $z$-axis.

The first order gyrotropic corrections to the far field are found from section $2.3 \mathrm{~b}$ and turn out to be [Gross, 1964]:

$$
\begin{aligned}
E_{\theta G} & =\frac{-i \omega \mu_{0}}{4 \pi} \sin \psi \\
E_{\psi G} & =\frac{i \omega \mu_{0}}{4 \pi} \cos \theta \cos \psi \\
H_{\theta G} & =\frac{e^{i k r}}{4 \pi} \cos \theta \cos \psi \\
H_{\psi G} & =\frac{-i k}{4 \pi} \sin \psi \\
r & \frac{e^{i k r}}{r} \\
E_{r G} & =H_{r G}=0
\end{aligned}
$$


where

$$
\mathscr{F}=\frac{k}{Y} \frac{e^{-i 2 k\left(z^{\prime}-z_{b}\right) \cos \theta}}{W_{1}^{\prime}\left(z_{b}\right)+i k \cos \theta W_{1}\left(z_{b}\right)} \int_{z_{b}}^{\infty} d z^{\prime \prime}\left(1+a z^{\prime \prime}\right) e^{i \sqrt{k^{2}-k_{t}^{2}\left(z^{\prime \prime}-z_{b}\right)}} W_{1}^{\prime}\left(z^{\prime \prime}\right)
$$

To account for the modification due to (40), one may define a new reflection coefficient $\Gamma_{1}^{\prime}$,

$$
\Gamma_{1}^{\prime}=\Gamma^{\prime}-\digamma \sec \theta \tan \psi
$$

which highlights the skewing of the far field pattern with $\psi$. The power patterns are similarly modified by the gyrotropic correction [Gross, 1964].

The integral in (41) may be evaluated approximately with the assumption of slight losses, thereby effectively truncating the integration interval. When the rate of variation of $X$ with $z$ is large, it is found that

$$
\mathscr{F}=-\frac{a}{k Y(1+\cos \theta)^{3}} e^{-i 2 k\left(z^{\prime}-z_{b}\right) \cos \theta}
$$

from which

$$
E_{\theta G}=\frac{i \omega \mu_{0}}{4 \pi} \frac{e^{i k r}}{r} \frac{\sin \psi}{(1+\cos \theta)^{3}} e^{-i 2 k\left(z^{\prime}-z_{b}\right) \cos \theta}
$$

and similarly for other components. Details of this calculation, as well as of the more involved small slope approximation, will be presented in a subsequent paper. Although a large slope is admitted, the inequality $a<<k Y$ must be satisfied in order to retain the perturbation character of the gyrotropic correction. Graphs of the angle dependent parameters of $E_{\theta u}, \vec{E}_{\theta G}$ and $E_{\psi G}$ are shown in figures 3 and 4.

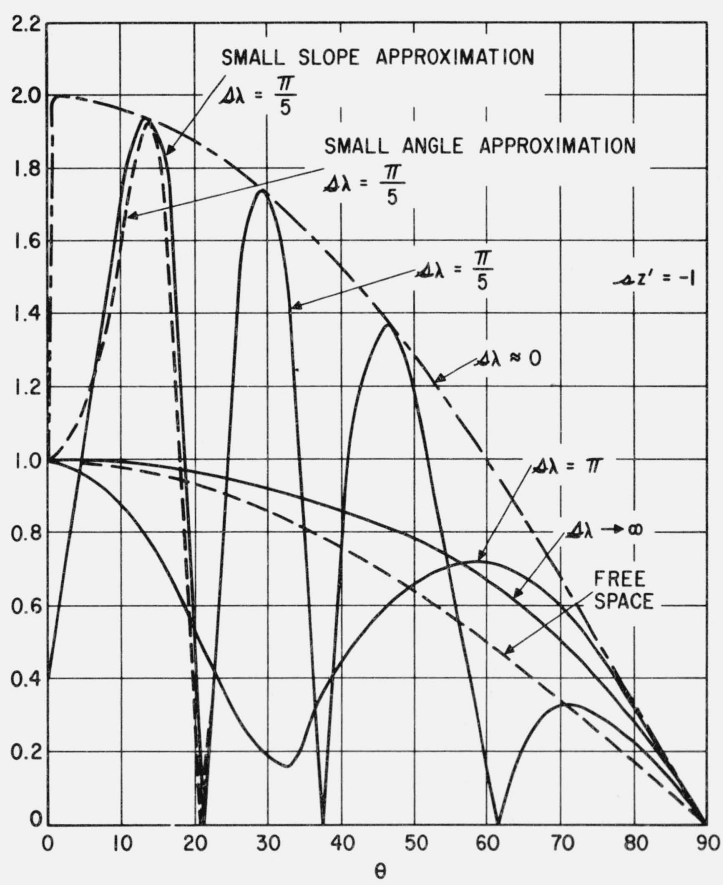

Figure 3. Uniaxial field component for the semi-infinite linear model.

The ordinate represents $\left|E_{\theta u}\right|$ in the $x z$ plane multiplied by the factor $\frac{4 \pi r}{\omega \mu_{0}}$,

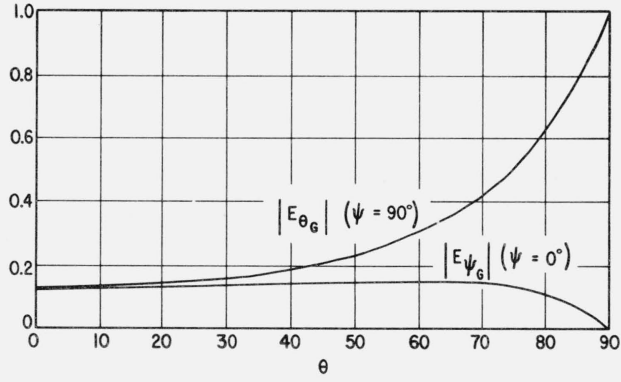

FIGURE 4. Relative gyrotropic corrections to field components.

The ordinate represents the pertinent field quantity, $\left|E_{\theta G}\right|$ or $\left|E_{\psi G}\right|$, multiplied by $\left(\frac{4 \pi \tilde{r} r}{\omega \mu_{0}}\right)\left(\frac{k Y}{a}\right)$, the abscissa $\theta$. 


\section{Discussion}

Figure 3 contains graphs descriptive of $\left|E_{\theta u}\right|$ versus $\theta$ in the $x z$ plane $\left(\psi=0^{\circ}\right)$ for various values of the normalized slope parameter $\lambda$, where $\lambda$ is the free-space wavelength. The curves represent the angular function of $E_{\theta u}$ in (38), with the source taken at the boundary $\left(a z^{\prime}=-1\right)$. Values of $a \lambda$ were selected to demonstrate different characteristics. The $\Delta \lambda \approx 0$ curve corresponds to the envelopes of the peaks of the multilobe pattern obtained for small values of $\Delta \lambda$. The $a \lambda=\pi / 5$ curve exhibits a four lobe pattern. This curve was plotted by using an approximation for $\Gamma^{\prime}$ valid when the rate of density variation in the medium is small, and contains nulls that go to zero. For comparison, a better approximation for the region near $\theta=0$ is shown by a dashed curve. The $a \lambda=\pi$ curve was plotted using exact values of the Whittaker functions in the expression for $\Gamma^{\prime}$. The curve has two lobes and a broader null that does not dip to zero. As a $\lambda$ increases further the lobes disappear and the pattern approaches that shown for $a \lambda \rightarrow \infty$. The free space curve is also shown for comparison with the $a \lambda \rightarrow \infty$ curve.

The multilobe patterns which are found for small values of the slope parameter are typical of highly reflective media. The pattern for an infinite value of the parameter is similar to the freespace curve shown in the figure, and is typical of a weakly reflecting region. Even when the source is moved away from the boundary these characteristics are basically unaltered [Gross, 1964]. The curves show, therefore, that more reflection is obtained for the small slope case, whereas regions with large slope reflect only a small amount of energy; the rest penetrates deeper into the medium. These results have also been examined in detail from a physical standpoint, for example, using ray theory [Gross, 1964], and have been found consistent with such viewpoints.

Figure 4 illustrates the variation of the angular factors of $\left|E_{\theta G}\right|$ and $\left|E_{\psi G}\right|$ with $\theta$ in their plane of maximum magnitude, $\psi=90^{\circ}$. These curves are plotted for the large slope approximation and are independent of the source point $z^{\prime}$, since $z^{\prime}$ enters only in an imaginary exponential factor. The phase varies with $z^{\prime}$, producing interference or enhancement of the uniaxial field components. $\left|E_{\theta G}\right|$ reaches a maximum at $\theta=90^{\circ}$, whereas, $\left|E_{\psi G}\right|$ is almost independent of the angle for $\theta<80^{\circ}$ and decreases to zero beyond. The magnitudes shown in the figure are only relative, since the absolute numerical contribution depends on the value of $Y$, which is considered suitably large for the purpose here.

Although $\left|E_{\theta G}\right|$ reaches a maximum at $\theta=90^{\circ}$, it can be shown [Gross, 1964] that the firstorder gyrotropic power is zero at this angle. In fact the Poynting vector is zero throughout the $x z$ and $y z$ planes where the only contributions from the gyrotropic corrections must be from higher order terms. It can also be shown that the gyrotropic power peaks in the $\psi=45^{\circ}, 135^{\circ}$ planes and is independent of $z^{\prime}$. With respect to $\theta$, the power is found to peak at $\theta=77^{\circ}$ for large values of $s \lambda$. These results indicate the skewness of the gyrotropic corrections.

\section{References}

Arbel, E., and L. B. Felsen (1963), Radiation from sources in anisotropic media. Parts I and II. Electromagnetic theory and antennas, ed. E. C. Jordan (Pergamon Press, New York, N.Y.).

Budden, K. G. (1961), Radio waves in the ionosphere. (Cambridge University Press, London).

Clemmow, P. C., and J. Heading (1954), Coupled forms of the differential equations governing radio propagation in the ionosphere, Proc. Cambridge Phil. Soc. 50, 319.

Felsen, L. B. (1964), Propagation and diffraction in uniaxially anisotropic regions. Part I. Theory. Part II. Applications, Proc. IEE (London) 111, No. 3, 445-464.

Felsen, L. B., and N. Marcuvitz (1956), Modal analysis and synthesis of electromagnetic fields, Research Report R-446-55(a) and (b), Microwave Research Institute, Polytechnic Institute of Brooklyn, Brooklyn, New York, N.Y.

Felsen, L. B., and N. Marcuvitz (1959), Modal analysis and synthesis of electromagnetic fields, Research Report R-726-59, Microwave Research Institute, Polytechnic Institute of Brooklyn, Brooklyn, N.Y.

Forsterling, K. (1942), Uber die Ausbreitung elektromagnetischer Wellen in einem magnetisierten Medium bei senkrechter Inzidenz, Hochfr. Elek. 59, 110.

Gross, S. H. (1964), Propagation in nonuniform, gyrotropıc media. Doctoral dissertation, Polytechnic Institute of Brooklyn, Brooklyn, N.Y.

Hougardy, R. W. (1961), A modified WKB approximation and its application to wave propagation. Doctoral dissertation (Physics), University of Calif., Los Angeles. 
Keller, H. (1953), Ionospheric propagation of plane waves, Report EM-56, Institute of Mathematical Sciences, New York University.

Slater, L. J. (1960), Confluent hypergeometric functions (Cambridge University Press, London).

\section{Additional Related References}

Epstein, P. S. (1930), Reflection of waves in an inhomogeneous absorbing medium, Proc. Natl. Acad. Sci. U.S. $16,627$. Hirsch, P. (1964), E-mode propagation in a plane-stratified plasma. Doctoral dissertation, Polytechnic Institute of Brooklyn, Brooklyn, N.Y.

(Paper 69D3-469) 